\title{
Uniqueness of Monotone Mono-stable Waves for Reaction-Diffusion Equations with Time Delay
}

\author{
W. Huang ${ }^{1 *}$, M. Han ${ }^{2}$ and M. Puckett ${ }^{1}$ \\ ${ }^{1}$ Department of Mathematical Sciences, University of Alabama in Huntsville \\ Huntsville, AL 35899, USA \\ ${ }^{2}$ Department of Mathematics, Sanghai Normal University \\ Shangai, China
}

\begin{abstract}
Many models in biology and ecology can be described by reaction-diffusion equations wit time delay. One of important solutions for these type of equations is the traveling wave solution that shows the phenomenon of wave propagation. The existence of traveling wave fronts has been proved for large class of equations, in particular, the monotone systems, such as the cooperative systems and some competition systems. However, the problem on the uniqueness of traveling wave (for a fixed wave speed) remains unsolved. In this paper, we show that, for a class of monotone diffusion systems with time delayed reaction term, the mono-stable traveling wave font is unique whenever it exists.
\end{abstract}

Key words: reaction-diffusion equations, time delay, traveling waves, monotone systems AMS subject classification: 34K99, 35K57, 92B99

\section{Introduction}

A reaction-diffusion equations with time delay has occurred frequently in literature as models for many population, ecological or biological problems. A general form of the equations can be

${ }^{*}$ Corresponding author. E-mail: huang@math.uah.edu 
expressed as

$$
\frac{\partial u(x, t)}{\partial t}=\mathbf{d} \Delta u(x, t)+F\left(u(x, t), \int_{-r}^{0} d \mu(\theta) u(x, t+\theta)\right),
$$

where $u=u(x, t) \in \mathbb{R}^{n}, \mathbf{d}=\operatorname{diag}\left(d_{1}, \cdots, d_{n}\right)$ is an $n \times n$ nonnegative diagonal matrix with at least one $d_{j}>0, x \in \mathbb{R}^{m}$ is the spatial variable, $t \geq 0$ is the time, $\Delta=\sum_{j=1}^{m} \frac{\partial^{2}}{\partial x_{j}^{2}}$ is the Laplace operator, $F \in C^{2}\left(\mathbb{R}^{n}, \mathbb{R}^{n}\right)$, and $\mu:[-r, 0] \rightarrow \mathbb{R}^{n} \times \mathbb{R}^{n}$ is of bounded variation. For the formulation and application of equations to practical problems we refer readers to [8]. The main interest of this paper is the traveling wave solutions of (1.1). Although the problem on the existence of traveling wave solutions for a general equation of (1.1) remains open, the existence of traveling waves for certain classes, in particular, for the monotone class of (1.1), has been proved $[3,5,7,9]$ (The existence of mono-stable traveling waves for a general system of monotone class of (1.1) has not been given in literature. However the existence of monotone mono-stable traveling wave can be proved using a monotone iteration approach similar to one for the monotone system without time delay.) The purpose of this paper is not to address the problem on the existence, but the uniqueness of traveling wave solution when (1.1) is a monotone system. The uniqueness of a traveling wave solution is important in application to practical problems. For instance, when using (1.1) to model many biological or physics problems, it is often the case that we search for a traveling wave connecting the equilibrium points $E_{1}$ and $E_{2}$, where the lower equilibrium $E_{1}=0$. If only the nonnegative solutions (the biologically or physically meaningful solutions) are considered, then it can be shown that, for a monotone system, the uniqueness of a monotone traveling wave actually implies the uniqueness of a nonnegative traveling wave (with respect to a fixed wave speed). For the simplest equation, i.e, the scalar Fisher equation, the uniqueness of the monotone traveling wave can be proved by analyzing a planar system. The uniqueness of monotone traveling waves also has been proved for some classes of scalar nonlocal types of reaction-diffusion equations $[2,6]$ using sophisticated analysis. Most recently, the uniqueness of monotone traveling waves for higher dimensional systems without time delay has been proved [5] by using the comparison argument and asymptotical behavior of solutions as time goes to $\pm \infty$. It turns out that the technique developed in [5] can also be applied to a time delayed monotone system (1.1). In this paper we shall prove that, for a class of time delayed reaction-diffusion systems (1.1), the monotone traveling wave solution is indeed unique whenever it exists.

Recall that (1.1) is a monotone system if

[A1 ] For $(u, v) \in \mathbb{R}^{n \times n}$,

$$
\frac{\partial F_{i}(u, v)}{\partial u_{j}} \geq 0, \quad i \neq j, \quad \frac{\partial F_{i}(u, v)}{\partial v_{j}} \geq 0 \quad \text { for } i, j,
$$

and $\mu_{i j}(\theta)$ is monotone increasing for all $i, j$, where $\mu(\theta)=\left[\mu_{i j}(\theta)\right]_{n \times n}$.

Throughout of this paper we suppose that [A1] holds. A typical example of equations that satisfies the condition [A1] is the cooperative system, in which an increase of one group's population will be beneficial to the population growth of all other groups. 
The reaction equation corresponding to (1.1) is the delay differential equation

$$
\dot{u}(t)=F\left(u(t), \int_{-r}^{0} d \mu(\theta) u(t+\theta)\right) .
$$

In addition to $[\mathbf{A 1}]$, we suppose that

[A2 ] System (1.2) has two constant equilibrium points $E_{i} \in \mathbb{R}^{n}$, such that $E_{1} \ll E_{2}, E_{1}$ is unstable, and $E_{2}$ is stable. (For the notation "«" see the definition right after Definition 2.2 in Section 2.)

Condition [A2] holds in many models. For instance, the SIS model in the epidemiology, if the reductive number $R_{0}>1$, has a disease free equilibrium $E_{1}=0$ which is unstable and a strictly positive endemic equilibrium that is stable. Another example is the dimensionless time delayed Lotka-Volterra compitition model

$$
\begin{aligned}
& u_{t}(x, t)=\Delta u(x, t)+u(x, t)\left[1-u(x, t)-a_{1} \int_{-r}^{0} d \mu_{i}(\theta) v(x, t+\theta)\right] \\
& v_{t}(x, t)=d \Delta v(x, t)+r v(x, t)\left[1-a_{2} \int_{-r}^{0} d \mu_{2}(\theta) u(x, t+\theta)-v(x, t)\right],
\end{aligned}
$$

where both $\mu_{1}$ and $\mu_{2}$ are increasing functions with $\mu_{i}(0)-\mu_{i}(-r)=1$. The corresponding reaction system is

$$
\begin{aligned}
& \dot{u}(t)=u(t)\left[1-u(t)-a_{1} \int_{-r}^{0} d \mu_{i}(\theta) v(t+\theta)\right], \\
& \dot{v}(t)=r v(t)\left[1-a_{2} \int_{-r}^{0} d \mu_{2}(\theta) u(t+\theta)-v(t)\right],
\end{aligned}
$$

which has always two boundary equilibrium points $\hat{E}_{1}=(0,1)$ and $\hat{E}_{2}=(1,0)$. It can be easily verified that, if $a_{1}<1<a_{2}$, then $\hat{E}_{1}$ is unstable and $\hat{E}_{2}$ is stable. System (1.3) can be transformed to a monotone system by introducing the variable $w=1-v$. Consequently, $\hat{E}_{1}$ and $\hat{E}_{2}$ are transformed respectively to equilibrium points $E_{1}=(0,0)$ and $E_{2}=(1,1)$ of the resulting monotone system with $E_{1}$ unstable and $E_{2}$ stable.

We further suppose that

[A3 ] Both the matrices $A_{i}+B_{i} \int_{-r}^{0} d \mu(\theta), i=1,2$, are irreducible, where

$$
A_{i}=D_{u} F\left(E_{i}, \int_{-r}^{0} d \mu(\theta) E_{i}\right), \quad B_{i}=D_{v} F\left(E_{i}, \int_{-r}^{0} d \mu(\theta) E_{i}\right)
$$

here $D_{u} F(u, v)$ and $D_{v} F(u, v)$ stand for partial derivatives of $F$ with respect to the variables $u$ and $v$, respectively.

In this paper we will establish the following theorem on the uniqueness of traveling wave solutions. 
Theorem 1..1. Under Assumptions [A1] - [A3], for each $c>0$, if (1.1) has a monotone traveling wave solution $u(x, t)=U(\nu \cdot x+c t)$ connecting $E_{1}$ and $E_{2}$, then the function $U(s), s=\nu \cdot x+c t$, is unique up to a time translation, where $\nu \in \mathbb{R}^{m}$ is a unit vector.

We remark that Condition [A3] is more technical that makes the analysis of the spectral properties easier. For certain class of equations this condition can be dropped. A complete proof of Theorem 1.1 will be given in Section 4. We first study an eigenvalue problem in Section 2. In Section 3 we use the information on the eigenvalues and eigenvectors to investigate the asymptotical behavior of a traveling wave solution as it converges to an equilibrium point. The results established in Sections 2 and 3 will be used in the proof of Theorem 1.1.

\section{An eigenvalue problem}

We will see that the asymptotical behavior of a traveling wave solution plays an important role in proving the unqueness. To understand the asymptotical behavior of a traveling wave when it converges to an equilibrium, we must study the eigenvalues and the eigenvectors of an associated linear system.

Definition 2..1. For a second order system of linear differential equations

$$
c \dot{v}(t)=\mathbf{d} \ddot{v}(t)+A v(t)+B \int_{-r}^{0} d \mu(\theta) v(t+c \theta),
$$

we say that $\lambda \in \mathbb{C}$ is an eigenvalue and $0 \neq \eta \in \mathbb{C}^{n}$ is the associated eigenvector corresponding to (2.1) if $\lambda$ and $\eta$ satisfy the equation

$$
\left[A+\lambda^{2} \mathbf{d}+B \int_{-r}^{0} d \mu(\theta) e^{\lambda c \theta}\right] \eta=c \lambda \eta
$$

It is obvious that $\lambda$ is an eigenvalue with the associated eigenvector $\eta$ if and only if (2.1) has a nonzero solution $v(t)=e^{\lambda t} \eta$.

Definition 2..2. We say that $\lambda$ is a simple eigenvalue of (2.1) if there is only one eigenvector associated to $\lambda$. Moreover, Eq.(2.1) has no solution of the form $v(t)=\left(t h_{1}+h_{2}\right) e^{\lambda t}$, where $h_{1}, h_{2} \in \mathbb{R}^{n}$ with $h_{1} \neq 0$.

In this paper, a nonnegative vector $h \in \mathbb{R}^{n}$ is denoted by $h \geq 0$. We use $h>0$ to indicate that $h$ is nonnegative and nonzero. We say that $h$ is strictly positive, denoted by $h \gg 0$, if all components of $h$ are positive. A real matrix is called nonnegative if all its entries are nonnegative. A nonnegative matrix is positive if it has at least one positive entry. The following lemma is well known for a positive matrix [1].

Lemma 2..1. Let $B=\left[b_{i j}\right] \in \mathbb{R}^{n \times n}$ be a positive and irreducible matrix with $b_{i i}>0$ for $i=$ $1, \cdots, n$. If $0<h \in \mathbb{R}^{n}$ and $h$ has $k(k<n)$ positive components, then Bh has at least $k+1$ positive components. 
Lemma 2..2. Let $c>0$ and $A, B \in \mathbb{R}^{n \times n}$ with the off diagonal entries of $A$ being nonnegative and $B$ being a nonnegative matrix. Moreover we suppose that the matrix $A+B \int_{-r}^{0} d \mu(\theta)$ is irreducible. Then

(i) If $\lambda \in \mathbb{R}$ is an eigenvalue and the associated eigenvector $\eta$ (with respect to (2.1)) is nonnegative, then (al) $\eta \gg 0$ and $\eta$ is the only associated eigenvector (up to a scalar multiplication); (a2) for $0 \neq \beta \in \mathbb{R}, \lambda+i \beta$ is not an eigenvalue.

(ii) If in addition (2.1) is stable (i.e. all eigenvalues of (2.1) have negative real part), then there is a unique $\lambda_{*} \leq 0$ such that the associated eigenvector $\eta$ with respect to (2.1) is strictly positive (up to a scalar multiplication). Moreover, $\lambda_{*}$ must be negative and simple.

Proof. Pick a sufficiently large number $\alpha$ such that $\alpha+a_{i i}>0$ for $i=1, \cdots, n$. Then the matrix $A+B \int_{-r}^{0} d \mu(\theta) e^{\lambda c \theta}+\alpha I+\lambda^{2} \mathbf{d}$ is a positive irreducible matrix. Since

$$
\left[A+B \int_{-r}^{0} d \mu(\theta) e^{\lambda c \theta}+\lambda^{2} \mathbf{d}\right] \eta=c \lambda \eta
$$

is equivalent to

$$
\left[A+B \int_{-r}^{0} d \mu(\theta) e^{\lambda c \theta}+\alpha I+\lambda^{2} \mathbf{d}\right] \eta=(c \lambda+\alpha) \eta,
$$

the theory for positive matrices implies that $\eta \gg 0$ and the eigenvector is unique. This proves (a1). For (a2), suppose the opposite, i.e. there is a $\beta \neq 0$ and $\xi \in \mathbb{C}$ such that

$$
\left[A+B \int_{-r}^{0} d \mu(\theta) e^{(\lambda+i \beta) c \theta}+(\lambda+i \beta)^{2} \mathbf{d}\right] \xi=c(\lambda+i \beta) \xi
$$

Then

$$
\left[A+B \int_{-r}^{0} d \mu(\theta) e^{(\lambda+i \beta) c \theta}+\alpha I+(\lambda+i \beta)^{2} \mathbf{d}\right] \xi=[c(\lambda+i \beta)+\alpha] \xi .
$$

A straightforward computation yields that

$$
\left[A+B \int_{-r}^{0} d \mu(\theta) e^{(\lambda+i \beta) c \theta}+\alpha I+\lambda^{2} \mathbf{d}\right] \xi=\left[(c \lambda+\alpha) I+\beta^{2} \mathbf{d}+i(c \beta I-2 \lambda \beta \mathbf{d})\right] \xi .
$$

Let $r(M)$ denote the spectral radius of the matrix $M$. Then (2.2) yields that

$$
c \lambda+\alpha=r\left(A+B \int_{-r}^{0} d \mu(\theta) e^{\lambda c \theta}+\alpha I+\lambda^{2} \mathbf{d}\right)>0 .
$$

Since $\mathbf{d}$ is a nonnegative diagonal matrix, we immediately deduce that

$$
\left[A+B \int_{-r}^{0} d \mu(\theta) e^{\lambda c \theta}+\alpha I+\lambda^{2} \mathbf{d}\right] \xi^{*}>(\alpha+c \lambda) \xi^{*}
$$


where $\xi^{*}=\left(\left|\xi_{1}\right|, \cdots,\left|\xi_{n}\right|\right)$ is a positive vector. The irreducibility therefore implies that

$$
r\left(A+B \int_{-r}^{0} d \mu(\theta) e^{\lambda c \theta} \alpha I+\lambda^{2} \mathbf{d}\right)>c \lambda+\alpha,
$$

contradicting (2.2).

To prove Part (ii), for real $\lambda$, we define the matrix

$$
M(\lambda)=A+B \int_{-r}^{0} d \mu(\theta) e^{\lambda c \theta}+\lambda^{2} \mathbf{d}+\alpha I .
$$

Then $M(\lambda)$ is a positive and irreducible matrix. Consider the equation

$$
M(\lambda) h=(\alpha+c \lambda) h .
$$

By the theory of positive matrices we know that this equation has a solution $h>0$ if and only if

$$
r(M(\lambda))=\alpha+c \lambda .
$$

It is apparent that $r(M(\lambda))$ is continuous with respect to $\lambda$. Let us consider $\lambda \leq 0$ such that the above equality holds. First we note that if $\lambda_{1}<\lambda_{2} \leq 0$, then $M\left(\lambda_{1}\right) \geq M\left(\lambda_{2}\right)$, which implies

$$
r\left(M\left(\lambda_{1}\right)\right) \geq r\left(M\left(\lambda_{2}\right)\right) \text {. }
$$

That is, $r(M(\lambda))$ is increasing as $\lambda \leq 0$ decreases. It is obvious that

$$
p(\alpha)=\alpha+c \lambda
$$

is decreasing as $\lambda$ decreases. Since (2.1) is stable, the dominant eigenvalue $\lambda_{0}$ of (2.1) is negative and the corresponding eigenvector $\zeta$ is positive. Thus we have

$$
M(0) \zeta=\left[A+B \int_{-r}^{0} d \mu(\theta) I+\alpha I\right] \zeta=\left(\alpha+\lambda_{0}\right) \zeta .
$$

It follows that

$$
r(M(0))=\alpha+\lambda_{0}<\alpha=p(\alpha) .
$$

On the other hand, we have $r(M(\lambda)) \geq 0$ for all $\lambda$ and $p(\alpha)<0$ for $\lambda<-\alpha / c$. Therefore, there is a unique $\lambda_{*}<0$ such that

$$
r\left(M\left(\lambda_{*}\right)\right)=\alpha+c \lambda_{*}
$$

Consequently, there is a unique strictly positive vector $\eta \in \mathbb{R}^{n}$ such that

$$
M\left(\lambda_{*}\right) \eta=\left(\alpha+c \lambda_{*}\right) \eta .
$$

Or equivalently,

$$
\left[A+B \int_{-r}^{0} d \mu(\theta) e^{\lambda_{*} c \theta}+\lambda_{*}^{2} \mathbf{d}\right] \eta=c \lambda_{*} \eta .
$$

It is more technical to prove that $\lambda_{*}$ is simple. To reduce the length of the paper we shall omit the detailed proof and refer the readers to the proof of Proposition 3.6 in [p.104, 4]. The argument used there can be directly applied to showing that $\lambda_{*}$ is a simple eigenvalue. 


\section{Asymptotical behavior of solutions to a non-autonomous lin- ear system}

In this section we use the result established in Section 2 to investigate the asymptotical behavior of solutions to a linear nonautonomous system. The asymptotical behavior of a solution as it converges to an equilibrium point is crucial to prove the uniqueness of a traveling wave solution.

Throughout the paper, an $n \times n$ matrix function $A(t)=\left[a_{i j}(t)\right], t \in \mathbb{R}$, is called an ENNmatrix function (essentially nonnegative matrix function) if $A(t)$ is bounded, continuous, and for all $i \neq j$,

$$
a_{i j}(t) \geq 0, \quad t \in \mathbb{R} .
$$

Lemma 3..1. Let $A(t)$ be an ENN-matrix function, $B(t)$ be a bounded and nonnegative matrix function, and let $w(t)$ be a nonzero, nonnegative, and bounded solution of the equation

$$
c \dot{w}(t)=\mathbf{d} \ddot{w}(t)+A(t) w(t)+B(t) \int_{-r}^{0} d \mu(\theta) w(t+c \theta), \quad t \in \mathbb{R} .
$$

Then the following hold.

(1) There is a real number $b>0$ such that if $d_{j}>0$, then

$$
-b w_{j}(t) \leq \dot{w}_{j}(t) \leq b w_{j}(t), \quad t \in \mathbb{R}
$$

where $w_{j}$ is the jth component of $w$. Consequently, we have

$$
\begin{aligned}
& w_{j}(s) \geq\left[w(t) e^{-b t}\right] e^{b s}, \quad s \leq t, \\
& w_{j}(t) \geq\left[w(s) e^{b s}\right] e^{-b t}, \quad t \geq s .
\end{aligned}
$$

In particular, $w_{j}\left(t_{0}\right)>0$ for some $t_{0}$ implies that $w_{j}(t)>0$ for all $t \in \mathbb{R}$.

(2) There is a real number $\delta>0$ such that if $d_{i}=0$, then

$$
w_{i}(t) \geq\left[w_{i}(s) e^{\delta s}\right] e^{-\delta t} \text { for all } t \geq s .
$$

In particular, $w_{i}\left(t_{0}\right)>0$ implies that $w_{i}(t)>0$ for all $t \geq t_{0}$.

(3) If $w \not \equiv 0$ and $A(\infty)=A_{2}$ and $B(\infty)=B_{2}$, then there exists a $t^{*}$ such that $w(t) \gg 0$ for all $t \geq t^{*}$.

(4) If $A(-\infty)=A_{1}, B(-\infty)=B_{1}$, and there exists a $t^{*}$ such that $w(t) \gg 0$ for all $t \geq t^{*}$, then $w(t) \gg 0$ for all $t \in \mathbb{R}$.

(5) If $A(-\infty)=A_{1}$ and $A(\infty)=A_{2}, B(\infty)=B_{2}$ and $B(-\infty)=B_{1}$, then $w \not \equiv 0$ implies $w(t) \gg 0$.

Here the matrices $A_{i}, B_{i}$ are defined in [A3] in Section 1. 
Proof. Let $\alpha$ be a sufficiently large number such that for $i=1, \cdots, n$,

$$
\alpha+a_{i i}(t) \geq 1, \quad t \in \mathbb{R} .
$$

Then $\alpha I+A(t)$ is a positive matrix for all $t \in \mathbb{R}$. For $d_{j}>0$, we rewrite the equation for $w_{j}$ as

$$
d_{j} \ddot{w}_{j}(t)-c \dot{w}_{j}(t)-\alpha w_{j}(t)=-\left[(\alpha I+A(t)) w(t)+B(t) \int_{-r}^{0} d \mu(\theta) w(t+c \theta)\right]_{j},
$$

where $[y]_{j}$ denotes the $j$ th component of the vector $y \in \mathbb{R}^{n}$. Let

$$
\begin{aligned}
& \alpha_{j}=\frac{c-\sqrt{c^{2}+4 d_{j} \alpha}}{2 d_{j}}<0 \\
& \beta_{j}=\frac{c+\sqrt{c^{2}+4 d_{j} \alpha}}{2 d_{j}}>0
\end{aligned}
$$

Then $w_{j}(t)$ can be expressed by

$$
\begin{aligned}
w_{j}(t)= & \frac{1}{d_{j}\left(\beta_{j}-\alpha_{j}\right)} \int_{-\infty}^{t} e^{\alpha_{j}(t-s)}\left[(\alpha I+A(s)) w(s)+B(s) \int_{-r}^{0} d \mu(\theta) w(s+c \theta)\right]_{j} d s \\
& +\frac{1}{d_{j}\left(\beta_{j}-\alpha_{j}\right)} \int_{t}^{\infty} e^{\beta_{j}(t-s)}\left[(\alpha I+A(s)) w(s)+B(s) \int_{-r}^{0} d \mu(\theta) w(s+c \theta)\right]_{j} d s .
\end{aligned}
$$

Thus we have

$$
\begin{aligned}
\dot{w}_{j}(t)= & \frac{\alpha_{j}}{d_{j}\left(\beta_{j}-\alpha_{j}\right)} \int_{-\infty}^{t} e^{\alpha_{j}(t-s)}\left[(\alpha I+A(s)) w(s)+B(s) \int_{-r}^{0} d \mu(\theta) w(s+c \theta)\right]_{j} d s \\
& +\frac{\beta_{j}}{d_{j}\left(\beta_{j}-\alpha_{j}\right)} \int_{t}^{\infty} e^{\beta_{j}(t-s)}\left[(\alpha I+A(s)) w(s)+B(s) \int_{-r}^{0} d \mu(\theta) w(s+c \theta)\right]_{j} d s .
\end{aligned}
$$

Let

$$
b=\max \left\{\left|\alpha_{j}\right|, \beta_{j}: d_{j}>0\right\} .
$$

Note that $(\alpha I+A(s)) w(s)+B(s) \int_{-r}^{0} d \mu(\theta) w(s+c \theta) \geq 0$. By (3.4) and (3.5) one easily sees

$$
-b w_{j}(t) \leq \dot{w}_{j}(t) \leq b w_{j}(t), \quad t \in \mathbb{R}, \quad j=1, \cdots, n .
$$

From the last inequalities, we deduce that for any $s<t$,

$$
w_{j}(s) \geq\left[w_{j}(t) e^{-b t}\right] e^{b s}, \quad w_{j}(t) \geq\left[w_{j}(s) e^{b s}\right] e^{-b t} .
$$

This completes the proof of Part (1).

If $d_{i}=0$, then $w_{i}(t)$ satisfies the inequality 


$$
c \dot{w}_{i}(t)+\alpha w_{i}(t)=\left[(\alpha I+A(t)) w(t)+B(t) \int_{-r}^{0} d \mu(\theta) w(t+c \theta)\right]_{i} \geq 0 .
$$

Part (2) of the lemma follows immediately from the above inequality with $\delta=\alpha / c$.

Now we prove Part (3). By (1) and (2), for any $i$, either $w_{i} \equiv 0$ or there is a $t_{i}$ such that $w_{i}(t)>0$ for all $t \geq t_{i}$. Since $w \not \equiv 0$, there is at least one $i$ such that $w_{i}(t)>0$ for all $t \geq t_{i}$. Suppose the statement of Part (3) is false. Then, without loss of generality (otherwise by reordering the components of $w$ if necessary), we can suppose that there are $t^{*}>0$ and a positive integer $k<n$ such that

$$
\begin{aligned}
& w_{i}(t) \quad>0, \quad t \geq t^{*}, \quad i=1,2, \cdots, k, \\
& w_{j}(t) \equiv 0, \quad t \in \mathbb{R}, \quad j=k+1, \cdots, n .
\end{aligned}
$$

It is clear that the matrix

$$
\alpha I+A(\infty)+B(\infty) \int_{-r}^{0} d \mu(\theta) I=\alpha I+A_{2}++B_{2} \int_{-r}^{0} d \mu(\theta) I
$$

is positive and irreducible with diagonal entries strictly positive. So is for the matrix $\alpha I+A(t)+$ $B(t) \int_{-r}^{0} d \mu(\theta) I$ for all sufficiently large $t$ by the continuity. It therefore follows from (3.7) and Lemma 2.1 that there is a sufficiently large $t_{0}>t^{*}$ such that the vector

$$
\left(\alpha I+A\left(t_{0}\right)\right) w\left(t_{0}\right)+B\left(t_{0}\right) \int_{-r}^{0} d \mu(t h) w\left(t_{0}+c \theta\right)
$$

has at least $k+1$ positive components. That is, there is a $j$ with $k+1 \leq j \leq n$ such that

$$
\left[\left(\alpha I+A\left(t_{0}\right)\right) w\left(t_{0}\right)+B\left(t_{0}\right) \int_{-r}^{0} d \mu(\theta) w\left(t_{0}+c \theta\right)\right]_{j}>0 .
$$

If $d_{j}>0$, then (3.4) implies that

$$
w_{j}(t)>0, \quad t \in \mathbb{R} .
$$

If $d_{j}=0$, then

$$
\dot{w}_{j}(t)+\frac{\alpha}{c} w_{j}(t)=\frac{1}{c}\left[(\alpha I+A(t)) w(t)+B(t) \int_{-r}^{0} d \mu(\theta) w(t+c \theta)\right]_{j}
$$

implies that

$$
w_{j}(t)=e^{-\frac{\alpha}{c}\left(t-t_{0}\right)}\left(w_{j}\left(t_{0}\right)+\frac{1}{c} \int_{t_{0}}^{t} e^{\frac{\alpha s}{c}}\left[(\alpha I+A(s)) w(s)+B(s) \int_{-r}^{0} d \mu(\theta) w(s+c \theta)\right]_{j} d s\right)>0
$$

for all $t>t_{0}$. In either case we have a contradiction to (3.8).

For the proof of Part (4), recall that there is at least one $d_{j} \neq 0$. Hence, by the assumption, we have $w_{j}(t)>0$ for all $t \in \mathbb{R}$. Arguing in the same way as for the proof of Part (3), one is able to see that $w(t) \gg 0$ for all $t \in \mathbb{R}$.

Finally, it is obvious that Part (5) is a direct consequence of (3) and (4). 
Now for $y=\left(y_{1}, \cdots, y_{n}\right) \in \mathbb{R}^{n}$ we define

$$
\|y\|=\sum_{i=1}^{n}\left|y_{i}\right| .
$$

Lemma 3..2. Let $A(t)$ be an ENN-matrix function and $B(t)$ be a bounded, nonnegative matrix function. Suppose that $w: \mathbb{R} \rightarrow \mathbb{R}^{n}$ is a strictly positive and bounded function satisfying the equation

$$
c \dot{w}(t)=\mathbf{d} \ddot{w}(t)+A(t) w(t)+B(t) \int_{-r}^{0} d \mu(\theta) w(t+c \theta), \quad t \in \mathbb{R}
$$

where

$$
A(-\infty)=A_{1}, \quad B(-\infty)=B_{1}, \quad A(\infty)=A_{2}, \quad B(\infty)=B_{2} .
$$

Let

$$
\begin{aligned}
s_{*} & =\inf \left\{s: \lim _{t \rightarrow \infty}\|w(t)\| e^{-s t}=0\right\}, \\
\nu^{*} & =\sup \left\{\nu: \lim _{t \rightarrow-\infty}\|w(t)\| e^{-\nu t}=0\right\}
\end{aligned}
$$

and let

$$
\begin{aligned}
& \hat{w}(T, s)=\int_{0}^{\infty} e^{-s t} w(T+t) d t, \quad T \in \mathbb{R}, \quad s>s_{*}, \\
& \tilde{w}(T, \nu)=\int_{-\infty}^{0} e^{-\nu t} w(T+t) d t, \quad T \in \mathbb{R}, \quad \nu<\nu^{*} .
\end{aligned}
$$

Then there are sequences $T_{n} \rightarrow \infty, s_{n} \rightarrow s_{*}, T_{n}^{\prime} \rightarrow-\infty$, and $\nu_{n} \rightarrow \nu^{*}$ as $n \rightarrow \infty$ such that

$$
\lim _{n \rightarrow \infty} \frac{w\left(T_{n}\right)}{\left\|\hat{w}\left(T_{n}, s_{n}\right)\right\|}=0, \quad \lim _{n \rightarrow \infty} \frac{w\left(T_{n}^{\prime}\right)}{\left\|\tilde{w}\left(T_{n}^{\prime}, \nu_{n}\right)\right\|}=0
$$

Proof. By (1) of Lemma 3.1, for $d_{j}>0$,

$$
\begin{aligned}
& w_{j}(t) \geq w(0) e^{-b t}, \quad t \geq 0, \\
& w_{j}(s) \geq w(0) e^{b s}, \quad s \leq 0 .
\end{aligned}
$$

Hence both $s_{*}$ and $\nu^{*}$ are well defined real numbers. Since the proof of Lemma 3.2 for the functions $\hat{w}(T, s)$ and $\tilde{w}(T, \nu)$ are same, we shall only prove the lemma for the function $\hat{w}(T, s)$. First, the positive of $w(t)$ implies that either $\int_{0}^{\infty} e^{-s * t} w(t) d t$ converges or $\left\|\int_{0}^{\infty} e^{-s * t} w(t) d t\right\|=\infty$. If $\left\|\int_{0}^{\infty} e^{-s * t} w(t) d t\right\|=\infty$, then $\left\|\int_{0}^{\infty} e^{-s * t} w(T+t) d t\right\|=\infty$ for any $T>0$. Hence by the continuity there exist sequences $T_{n} \rightarrow \infty$ and $s_{*}<s_{n} \rightarrow s_{*}$ as $n \rightarrow \infty$ such that

$$
\left\|\hat{w}\left(T_{n}, s_{n}\right)\right\|=\left\|\int_{0}^{\infty} e^{-s_{n} t} w\left(T_{n}+t\right) d t\right\| \rightarrow \infty, \quad n \rightarrow \infty .
$$

Note that $w\left(T_{n}\right)$ is bounded, it immediately follows that

$$
\lim _{n \rightarrow \infty} \frac{w\left(T_{n}\right)}{\left\|\hat{w}\left(T_{n}, s_{n}\right)\right\|}=0 .
$$


Next suppose

$$
\left\|\int_{0}^{\infty} e^{-s_{*} t} w(t) d t\right\|<\infty
$$

Let

$$
\xi(t)=\|w(t)\| .
$$

Since $w(t)$ is positive, we have

$$
\hat{w}\left(T, s_{*}\right)\|=\| \int_{0}^{\infty} e^{-s_{*} t} w(T+t) d t \|=\int_{0}^{\infty} e^{-s_{*} t} \xi(T+t) d t
$$

and $\int_{0}^{\infty} e^{-s_{*} t} \xi(T+t) d t<\infty$ for all $T>0$. To complete the proof of Lemma 3.2, it is sufficient to show that

$$
\lim _{T \rightarrow \infty} \inf \frac{\xi(T)}{\int_{0}^{\infty} e^{-s_{*} t} \xi(T+t) d t}=0 .
$$

Suppose (3.13) does not hold. Then there exist positive number $\sigma$ and $T^{*}$ such that

$$
\xi(T) \geq \sigma \int_{0}^{\infty} e^{-s_{*} t} \xi(T+t) d t=\sigma e^{s_{*} T} \int_{T}^{\infty} e^{-s_{*} \theta} \xi(\theta) d \theta, \quad T \geq T^{*} .
$$

Without loss of generality we suppose $T^{*}=0$. Then (3.14) yields that

$$
e^{-s_{*} t} \xi(t) \geq \sigma \int_{t}^{\infty} e^{-s_{*} \theta} \xi(\theta) d \theta, \quad t \geq 0
$$

If follows from the above inequality that

$$
\frac{d}{d t} \ln \left(\int_{t}^{\infty} e^{-s_{*} \theta} \xi(\theta) d \theta\right)=-\frac{e^{-s_{*} t} \xi(t)}{\int_{t}^{\infty} e^{-s_{*} \theta} \xi(\theta) d \theta} \leq-\sigma .
$$

Integrating (3.15) from 0 to $t$ we obtain

$$
\ln \left(\frac{\int_{t}^{\infty} e^{-s_{*} \theta} \xi(\theta) d \theta}{\int_{0}^{\infty} e^{-s_{*} \theta} \xi(\theta) d \theta}\right) \leq-\sigma t
$$

or equivalently,

$$
\int_{t}^{\infty} e^{-s_{*} \theta} \xi(\theta) d \theta \leq K e^{-\sigma t}, \quad t>0
$$

with $K=\int_{0}^{\infty} e^{-s_{*} \theta} \xi(\theta) d \theta$. Let $\epsilon=\sigma / 2$. By exchanging the order of integration and with the use of (3.16), we deduce that

$$
\begin{aligned}
\frac{1}{\epsilon} \int_{0}^{\infty}\left[e^{-\left(s_{*}-\epsilon\right) \theta}-e^{-s_{*} \theta}\right] \xi(\theta) d \theta & =\int_{0}^{\infty} e^{-s_{*} \theta} \xi(\theta) \frac{1}{\epsilon}\left(e^{\epsilon \theta}-1\right) d \theta \\
& =\int_{0}^{\infty} e^{-s_{*} \theta} \xi(\theta) \int_{0}^{\theta} e^{\epsilon t} d t \\
& =\int_{0}^{\infty} e^{\epsilon t}\left[\int_{t}^{\infty} e^{-s_{*} \theta} \xi(\theta) d \theta\right] d t \\
& \leq K \int_{0}^{\infty} e^{\epsilon t} e^{-\sigma t} d t \\
& \leq K \int_{0}^{\infty} e^{-\epsilon t} d t \\
& <\infty
\end{aligned}
$$


Recall that $\int_{0}^{\infty} e^{-s_{*} t} \xi(t) d t<\infty$. (3.17) immediately implies that

$$
\int_{0}^{\infty} e^{-\left(s_{*}-\epsilon\right) t} \xi(t)<\infty
$$

Therefore, there must be a sequence $t_{n} \rightarrow \infty$ as $n \rightarrow \infty$ such that such that

$$
\lim _{n \rightarrow \infty} e^{-\left(s_{*}-\epsilon\right) t_{n}} \xi\left(t_{n}\right)=0 .
$$

By Part (1) of Lemma 3.1, for $d_{j}>0$, we have

$$
\left|\dot{w}_{j}(t)\right| \leq b\left|w_{j}(t)\right| \leq b\|w(t)\|, \quad t \in \mathbb{R} .
$$

Moreover, Parts (1) and (2) of Lemma 3.1 imply that for $k=\max \{b, \delta\}$,

$$
w(t+c \theta) \leq e^{k c r} w(t), \quad \theta \in[-r, 0], \quad t \in \mathbb{R} .
$$

It follows that, for $d_{i}=0$,

$$
\begin{aligned}
\left|\dot{w}_{i}(t)\right| & \leq \frac{1}{c}\left\|A(t) w(t)+B(t) \int_{-r}^{0} d \mu(\theta) w(t+\theta)\right\| \\
& \leq \frac{1}{c}\left(\|A(t)\|+\|B(t)\|\left\|\int_{-r}^{0} d \mu(\theta) I\right\| e^{k c r}\right)\|w(t)\| .
\end{aligned}
$$

Since $A(t)$ and $B(t)$ are bounded, (3.20) and (3.22) yield that there is a constant $M>0$ such that

$$
\|\dot{w}(t)\| \leq M\|w(t)\|=M \xi(t) .
$$

Therefore

$$
\left\|\int_{0}^{\infty} e^{-\left(s_{*}-\epsilon\right) \tau} \dot{w}(\tau) d \tau\right\| \leq \int_{0}^{\infty} e^{-\left(s_{*}-\epsilon\right) \tau} \xi(\tau) d \tau .
$$

So that $\int_{0}^{\infty} e^{-\left(s_{*}-\epsilon\right) \tau} \dot{w}(\tau) d \tau$ converges. Integrating $e^{-\left(s_{*}-\epsilon\right) \tau} \dot{w}(\tau)$ from $t$ to $t_{n}$ we obtain

$$
\begin{aligned}
\int_{t}^{t_{n}} e^{-\left(s_{*}-\epsilon\right) \tau} \dot{w}(\tau) d \tau= & e^{-\left(s_{*}-\epsilon\right) t_{n}} w\left(t_{n}\right)-e^{-\left(s_{*}-\epsilon\right) t} w(t) \\
& +\left(s_{*}-\epsilon\right) \int_{t}^{t_{n}} e^{-\left(s_{*}-\epsilon\right) \tau} w(\tau) d \tau .
\end{aligned}
$$

Letting $n \rightarrow \infty$ in (3.23) and noticing that $\left\|e^{-\left(s_{*}-\epsilon\right) t_{n}} w\left(t_{n}\right)\right\|=e^{-\left(s_{*}-\epsilon\right) t_{n}} \xi\left(t_{n}\right) \rightarrow 0$ as $n \rightarrow \infty$, we arrive at

$$
e^{-\left(s_{*}-\epsilon\right) t} w(t)=\left(s_{*}-\epsilon\right) \int_{t}^{\infty} e^{-\left(s_{*}-\epsilon\right) \tau} w(\tau) d \tau-\int_{t}^{\infty} e^{-\left(s_{*}-\epsilon\right) \tau} \dot{w}(\tau) d \tau
$$

From (3.24) we immediately conclude that

$$
e^{-\left(s_{*}-\epsilon\right) t} w(t) \rightarrow 0, \quad \text { as } \quad t \rightarrow \infty,
$$

which contradicts the definition of $s_{*}$. 
Lemma 3..3. Let $A(t)$ be an ENN-matrix function, $B(t)$ be bounded, nonnegative matrix function, and $w(t)$ be a strictly positive and bounded function satisfying

$$
c \dot{w}(t)=\mathbf{d} \ddot{w}(t)+A(t) w(t)+B(t) \int_{-r}^{0} d \mu(\theta) w(t+c \theta), \quad t \in \mathbb{R} .
$$

(a) Suppose that $A(t) \rightarrow A_{2}, B(t) \rightarrow B_{2}$, and $w(t) \rightarrow 0$ as $t \rightarrow \infty$. Then there is an $\gamma>0$ such that

$$
w(t)=\gamma \eta e^{\lambda_{*} t}+o\left(e^{\lambda_{*} t}\right) \quad \text { as } \quad t \rightarrow \infty .
$$

where $\lambda_{*}<0$ and $\eta \in \mathbb{R}^{n}$ is a strictly positive vector satisfying

$$
\left[A_{2}+B_{2} \int_{-r}^{0} d \mu(\theta) e^{\lambda_{*} c \theta} I+\lambda_{*}^{2} \mathbf{d}\right] \eta=c \lambda_{*} \eta
$$

[By Assumptions [A1], [A2], and Lemma 2.2, $\lambda_{*}<0$ and the strictly positive vector $\eta$ (up to a scalar multiplication) are uniquely determined.]

(b) Suppose that $A(t) \rightarrow A_{1}, B(t) \rightarrow B_{1}$, and $w(t) \rightarrow 0$ as $t \rightarrow-\infty$. Then there are a $\nu>0, a$ strictly positive vector $\xi \in \mathbb{R}^{n}$, and an integer $k \geq 0$ such that

$$
w(t)=(-1)^{k} t^{k} \xi e^{\nu t}+o\left(t^{k} e^{\nu t}\right) \quad \text { as } \quad t \rightarrow-\infty .
$$

Proof. First consider the case (a). Let

$$
s_{*}=\inf \left\{s: \lim _{t \rightarrow \infty}|w(t)| e^{-s t}=0\right\} .
$$

We write $w(t)$ as a solution of the equation

$$
\begin{aligned}
c \dot{w}(t) & =\mathbf{d} \ddot{w}(t)+A_{2} w(t)+B_{2} \int_{-r}^{0} d \mu(\theta) w(t+c \theta) \\
& +\left[A(t)-A_{2}\right] w(t)+\left[B(t)-B_{2}\right] \int_{-r}^{0} d \mu(\theta) w(t+c \theta), \quad t \in \mathbb{R} .
\end{aligned}
$$

Now for $T>0$ and $s>s_{*}$, the Laplace transform of $w(T+\cdot)$,

$$
\hat{w}(T, s)=\int_{0}^{\infty} w(T+t) e^{-s t} d t
$$

is well defined. Applying the Laplace transform to (3.25) with $s>s_{*}$ yields

$$
\begin{aligned}
-c w(T)+c s \hat{w}(T, s)= & -\mathbf{d} \dot{w}(T)-s \mathbf{d} w(T)+\left[s^{2} \mathbf{d}+A_{2}+B_{2} \int_{-r}^{0} d \mu(\theta) e^{s c \theta}\right] \hat{w}(T, s) \\
& +K(T, s),
\end{aligned}
$$

where 


$$
\begin{aligned}
K(T, s)= & \int_{0}^{\infty}\left[A(T+t)-A_{2}\right] w(T+t) e^{-s t} d t \\
& +\int_{0}^{\infty}\left[\left(B(T+t)-B_{2}\right) \int_{-r}^{0} d \mu(\theta) w(T+t+c \theta)\right] e^{-s t} d t \\
& +B_{2} \int_{-r}^{0} d \mu(\theta)\left[e^{s c \theta} \int_{c \theta}^{0} e^{-s \tau} w(T+\tau) d \tau\right] .
\end{aligned}
$$

By Lemma 3.2, there are sequences $\left\{T_{n}\right\}$ and $\left\{s_{n}\right\}$ such that

$$
T_{n} \rightarrow \infty, \quad s_{n}>s_{*}, \quad s_{n} \rightarrow s_{*} \quad \text { as } \quad n \rightarrow \infty
$$

and

$$
\frac{w\left(T_{n}\right)}{\left\|\hat{w}\left(T_{n}, s_{n}\right)\right\|} \rightarrow 0 \quad \text { as } \quad n \rightarrow \infty
$$

Moreover, from (3.27) and Part (1) of Lemma 3.1 it follows that for $d_{j} \neq 0$,

$$
\frac{\dot{w}_{j}\left(T_{n}\right)}{\left\|\hat{w}\left(T_{n}, s_{n}\right)\right\|} \rightarrow 0 \quad \text { as } \quad n \rightarrow \infty
$$

From Parts (1) and (2) of Lemma 3.1 it follows that there is a positive number $k>0$ such that

$$
w\left(T_{n}+t+c \tau\right) \leq w\left(T_{n}+t\right) e^{k c r}, \quad \tau \in[-r, 0] .
$$

The assumption

$$
\left\|A(t)-A_{2}\right\| \rightarrow 0, \quad\left\|B(t)-B_{2}\right\| \rightarrow 0 \quad \text { as } \quad t \rightarrow \infty
$$

yields that

$$
M_{n}=\sup \left\{\left\|A\left(T_{n}+t\right)-A_{2}\right\|,\left\|B\left(T_{n}+t\right)-B_{2}\right\|: t \geq 0\right\} \rightarrow 0 \quad \text { as } \quad n \rightarrow \infty
$$

Since

$$
\begin{aligned}
& \left\|\int_{0}^{\infty}\left[A\left(T_{n}+t\right)-A_{2}\right] w\left(T_{n}+t\right) e^{-s t} d t\right\| \leq M_{n} \int_{0}^{\infty}\left\|w\left(T_{n}+t\right)\right\| e^{-s t} d t \\
& =M_{n}\left\|\int_{0}^{\infty} w\left(T_{n}+t\right) e^{-s t} d t\right\| \\
& =M_{n}\|\hat{w}(T, s)\| \\
& \left\|\int_{0}^{\infty}\left[B\left(T_{n}+t\right)-B_{2}\right] \int_{-r}^{0} d \mu(\theta) w\left(T_{n}+t+c \theta\right) e^{-s t} d t\right\| \\
& \leq M_{n} \int_{0}^{\infty} \int_{-r}^{0} d \mu e^{\sigma c r}\left\|w\left(T_{n}+t\right)\right\| e^{-s t} d t \\
& =e^{k c r} M_{n}\left\|\int_{-r}^{0} d \mu(\theta) I\right\|\left\|\int_{0}^{\infty} w\left(T_{n}+t\right) e^{-s t} d t\right\| \\
& =e^{k c r} M_{n}\left\|\int_{-r}^{0} d \mu(\theta) I\right\|\|\hat{w}(T, s)\|,
\end{aligned}
$$




$$
\left\|B_{2} \int_{-r}^{0} d \mu(\theta) e^{s c \theta}\left[\int_{c \theta}^{0} e^{-s \tau} w\left(T_{n}+\tau\right) d \tau\right]\right\| \leq\left\|B_{2}\right\|\left\|\int_{-r}^{0} d \mu(\theta) e^{s c \theta} c \theta\right\| e^{k c r}\left\|w\left(T_{n}\right)\right\|
$$

from (3.27), (3.29) - (3.32) it follows that, as $n \rightarrow \infty$,

$$
\begin{aligned}
\frac{\left\|K\left(T_{n}, s_{n}\right)\right\|}{\left\|\hat{w}\left(T_{n}, s_{n}\right)\right\|} \leq & M_{n}\left(1+e^{k c r}\left\|\int_{-r}^{0} d \mu(\theta) I\right\|\right) \\
& +\left\|B_{2}\right\|\left\|\int_{-r}^{0} d \mu(\theta) e^{s_{n} c \theta} c \theta\right\| \frac{\left\|w\left(T_{n}\right)\right\|}{\left\|\hat{w}\left(T_{n}, s_{n}\right)\right\|} \\
\rightarrow & 0 .
\end{aligned}
$$

Moreover, (3.27) and (3.28) yield that

$$
\frac{-\mathbf{d} \dot{w}\left(T_{n}\right)-s_{n} \mathbf{d} w\left(t_{n}\right)}{\left\|\hat{w}\left(T_{n}, s_{n}\right)\right\|} \rightarrow 0 \quad \text { as } n \rightarrow \infty .
$$

It is obvious that $\left\{\hat{w}\left(T_{n}, s_{n}\right) /\left\|\hat{w}\left(T_{n}, s_{n}\right)\right\|\right\}$ has a convergent subsequence. Without loss of generality suppose

$$
\lim _{n \rightarrow \infty} \frac{\hat{w}\left(T_{n}, s_{n}\right)}{\left\|\hat{w}\left(T_{n}, s_{n}\right)\right\|}=\tilde{\eta}
$$

Then it is clear that $\tilde{\eta}>0$. Now dividing (3.26) by $\left\|\hat{w}\left(T_{n}, s_{n}\right)\right\|$ and letting $n \rightarrow \infty$, with the use of (3.26), (3.27), (3.32) and (3.33), we immediately obtain

$$
c s_{*} \tilde{\eta}=\left[s_{*}^{2} \mathbf{d}+A_{2}+B_{2} \int_{-r}^{0} d \mu(\theta) e^{s_{*} c \theta}\right] \tilde{\eta} .
$$

It therefore follows from Part (ii) of Lemma 2.2 that we must have

$$
s_{*}=\lambda_{*}<0 \quad \text { and } \quad \tilde{\eta}=\gamma \eta
$$

for some $\gamma>0$. The conclusion of Part (a) follows. The proof of Part (b) is essentially the same as above. Let

$$
\nu=\sup \left\{\tau: \lim _{t \rightarrow-\infty}|w(t)| e^{-\tau t}=0\right\} .
$$

Then $0 \leq \nu<\infty$. For $T<0$ and $\tau<\nu$, let

$$
\tilde{w}(T, \tau)=\int_{-\infty}^{0} w(T+t) e^{-\tau t} d t
$$

Then, by Lemma 3.2, there are sequences $\left\{T_{n}\right\}$ and $\left\{\tau_{n}\right\}$ such that

$$
T_{n} \rightarrow-\infty, \quad \tau_{n}<\nu, \quad \tau_{n} \rightarrow \nu \quad \text { as } \quad n \rightarrow \infty
$$


and

$$
\begin{aligned}
& \frac{w\left(T_{n}\right)}{\left\|\tilde{w}\left(T_{n}, \tau_{n}\right)\right\|} \rightarrow 0 \quad \text { as } \quad n \rightarrow \infty, \\
& \frac{\tilde{w}\left(T_{n}, \tau_{n}\right)}{\left\|\tilde{w}\left(T_{n}, \tau_{n}\right)\right\|} \rightarrow \xi>0 \quad \text { as } n \rightarrow \infty .
\end{aligned}
$$

Then argue in the same way for the function $\hat{w}(T, s)$ we deduce that

$$
c \nu \xi=\left[\nu^{2} \mathbf{d}+A_{1}+B_{1} \int_{-r}^{0} d \mu(\theta) e^{\nu c \theta}\right] \xi
$$

Thus $\xi$ must be strictly positive. Moreover, it is obvious that $\nu>0$ because the equilibrium $E_{1}$ is unstable with respect to the system (1.2). For this case, since $\nu$ may not be a simple eigenvalue, we can express $w(t)$ in the form given in Part (b).

\section{Proof of Theorem 1.1}

Proof. For a fixed $c>0$, suppose $u(x, t)=U_{1}(\nu \cdot x+c t)$ and $v(x, t)=U_{2}(\nu \cdot x+c t)$ are two monotone traveling wave solutions connecting the equilibria $E_{1}$ and $E_{2}$. Then $U_{i}$ satisfies the equation

$$
c \dot{U}_{i}(t)=\mathbf{d} \ddot{U}_{i}(t)+F\left(U_{i}(t), \int_{-r}^{0} d \mu(\theta) U_{i}(t+c \theta)\right), \quad t \in \mathbb{R}, \quad i=1,2 .
$$

To show the uniqueness it suffices to show that $U_{1}$ is a translation of $U_{2}$. That is, there is a constant $a^{*}$ such that

$$
U_{1}(t)=U_{2}\left(t+a^{*}\right), \quad t \in \mathbb{R} .
$$

For this purpose let us first establish the

Claim There are positive numbers $\gamma_{i}, \nu_{i}$ and strictly positive vectors $\xi_{i} \in \mathbb{R}^{n}$, and integers $k_{i} \geq 0$, such that for $i=1,2$,

$$
\begin{aligned}
& U_{i}(t)=E_{2}-\gamma_{i} e^{\lambda_{*} t} \eta+o\left(e^{\lambda_{*} t}\right), \quad \text { as } \quad t \rightarrow \infty, \\
& U_{i}(t)=E_{1}+(-1)^{k_{i}} t^{k_{i}} e^{\nu_{i} t} \xi_{i}+o\left(t^{k_{i}} e^{\nu_{i} t}\right), \quad \text { as } \quad t \rightarrow-\infty,
\end{aligned}
$$

where $\lambda_{*}<0$ and the vector $\eta$ are defined in Lemma 2.4 (a).

Proof of Claim For $i=1,2$, let $V_{i}(t)=E_{2}-U_{i}(t)$ for $t \in \mathbb{R}$. Then $V_{i}(t) \rightarrow 0$ as $t \rightarrow \infty$. A straightforward computation shows that $V_{i}(t)$ satisfies the equation

$$
c \dot{v}(t)=\mathbf{d} \ddot{v}(t)+A^{i}(t) v(t)+B^{i}(t) \int_{-r}^{0} d \mu(\theta) v(t+c \theta),
$$


where

$$
\begin{aligned}
& \left.A^{i}(t)=\int_{0}^{1} D_{u} F\left(E_{2}-(1-\tau)\right) V_{i}(t), \int_{-r}^{0} d \mu(\theta)\left(E_{2}-(1-\tau)\right) V_{i}(t+c \theta)\right) d \tau \\
& \left.B^{i}(t)=\int_{0}^{1} D_{v} F\left(E_{2}-(1-\tau)\right) V_{i}(t), \int_{-r}^{0} d \mu(\theta)\left(E_{2}-(1-\tau)\right) V_{i}(t+c \theta)\right) d \tau .
\end{aligned}
$$

Thus, by Assumption [A1], $A^{i}(t)$ is an ENN-matrix that converges to $A_{2}$ and $B^{i}(t)$ is nonnegative converging to $B_{2}$ as $t \rightarrow \infty$. Noting that $V_{i}(-\infty)=E_{2}-E_{1} \gg 0$, Lemma 3.1 (1) implies that $V_{i}(t)$ is strictly positive. It follows from Lemma 3.3 that

$$
E_{2}-U_{i}(t)=V_{i}(t)=\gamma_{i} e^{\lambda_{*} t} \eta+o\left(e^{\lambda_{*} t}\right), \quad \text { as } \quad t \rightarrow \infty
$$

for some $\gamma_{i}>0$. This proves (4.1).

To prove (4.2), let $W_{i}(t)=U_{i}(t)-E_{1}, i=1,2$. Then

$$
c \dot{W}_{i}(t)=\mathbf{d} \ddot{W}_{i}(t)+H^{i}(t) W_{i}(t)+K^{i}(t) \int_{-r}^{0} d \mu(\theta) W_{i}(t+c \theta)
$$

with

$$
\begin{aligned}
& H^{i}(t)=\int_{0}^{1} D_{u} F\left(E_{1}+\tau W_{i}(t), \int_{-r}^{0} d \mu(\theta)\left[E_{1}+\tau W_{i}(t+c \theta)\right]\right) d \tau, \\
& K^{i}(t)=\int_{0}^{1} D_{v} F\left(E_{1}+\tau W_{i}(t), \int_{-r}^{0} d \mu(\theta)\left[E_{1}+\tau W_{i}(t+c \theta)\right]\right) d \tau .
\end{aligned}
$$

It is clear that $W_{i}(t)$ is nonnegative. Moreover

$$
W_{i}(\infty)=U_{i}(\infty)-E_{1}=E_{2}-E_{1} \gg 0
$$

implies that there is a $t^{*} \in \mathbb{R}$ such that $W_{i}(t) \gg 0$ for all $t \geq t^{*}$. It is obvious that $H^{i}(-\infty)=A_{1}$ and $K^{i}(-\infty)=B_{1}$. Consequently we have $W_{i}(t) \gg 0$ for all $t \in \mathbb{R}$ by Lemma 3.1 (4). (4.2) therefore follows from Lemma 3.3 (b) .

Now we consider two cases.

Case $1 \nu_{1} \neq \nu_{2}$, or $\nu_{1}=\nu_{2}$ and $k_{1} \neq k_{2}$.

Without loss of generality, we suppose $\nu_{2}<\nu_{1}$, or $k_{2}>k_{1}$ if $\nu_{1}=\nu_{2}$. We define

$$
a^{*}=\inf \left\{a \in \mathbb{R}: U_{2}(t+a) \gg U_{1}(t), t \in \mathbb{R}\right\} .
$$

Let us show that $a^{*}$ is a real number. First we have

$$
U_{2}(-\infty)=E_{1} \ll U_{1}(0) .
$$

Hence, by continuity, there is an $a_{0}$ such that $U_{2}\left(a_{0}\right) \ll U_{1}(0)$. This implies that the set

$$
\Omega=\left\{a \in \mathbb{R}: U_{2}(t+a) \gg U_{1}(t), t \in \mathbb{R}\right\}
$$


is bounded below. Next, by the assumption and (3.2), we see that there is a $T_{1}<0$ such that

$$
U_{2}(t) \gg U_{1}(t) \text { for all } t \leq T_{1} \text {. }
$$

Noticing that $\lambda_{*}<0$, we can pick a number $a_{1}>0$ such that $\gamma_{2} e^{\lambda_{*} a_{1}}<\gamma_{1}$. Then by (3.1) we have

$$
U_{2}\left(t+a_{1}\right)-U_{1}(t)=\left[\gamma_{1}-\gamma_{2} e^{\lambda_{*} a_{1}}\right] e^{\lambda_{*} t} \eta+o\left(e^{\lambda_{*} t}\right) \quad \text { as } \quad t \rightarrow \infty .
$$

It follows that there is a $T_{2}>0$ such that

$$
U_{2}\left(t+a_{1}\right) \gg U_{1}(t), \quad t \geq T_{2} .
$$

Let $a_{2}=T_{2}-T_{1}+a_{1}$. Then, the above inequality and the monotonicity of $U_{1}$ and $U_{2}$ yield that for all $t \in\left[T_{1}, T_{2}\right]$, we have

$$
U_{2}\left(t+a_{2}\right) \geq U_{2}\left(T_{1}+a_{2}\right)=U_{2}\left(T_{2}+a_{1}\right) \gg U_{1}\left(T_{2}\right) \geq U_{1}(t) .
$$

Since $a_{2}>a_{1}$, from (3.3) and (3.4), it follows that

$$
U_{2}\left(t+a_{2}\right) \gg U_{1}(t), \quad t \in\left(-\infty, T_{1}\right] \cup\left[T_{2}, \infty\right) .
$$

Thus $a_{2} \in \Omega$ by (3.5) and (3.6). Hence $\Omega$ is nonempty and bounded below. This implies $a^{*}$ is a real number. By the definition of $a^{*}$ and the continuity of $U_{i}(t)$ for $i=1,2$, we conclude that

$$
U_{2}\left(t+a^{*}\right) \geq U_{1}(t), \quad t \in \mathbb{R} .
$$

We claim that $U_{2}\left(t+a^{*}\right) \equiv U_{1}(t)$. If this is not the case, let $w(t)=U_{2}\left(t+a^{*}\right)-U_{1}(t)$. Then $w(t) \geq 0$ and $w \not \equiv 0$. In addition, we have

$$
c \dot{w}(t)=\ddot{w}(t)+A(t) w(t)+B(t) \int_{-r}^{0} d \mu(\theta) W(t+c \theta),
$$

where

$$
\begin{aligned}
& A(t)=\int_{0}^{1} D_{u} F(\Phi(t, \tau), \Psi(t, \tau)) d \tau \\
& B(t)=\int_{0}^{1} D_{v} F(\Phi(t, \tau), \Psi(t, \tau)) d \tau
\end{aligned}
$$

with

$$
\begin{aligned}
& \Phi(t, \tau)=U_{1}(t)+\tau\left[U_{2}\left(t+a^{*}\right)-U_{1}(t)\right], \\
& \Psi(t, \tau)=\int_{-r}^{0} d \mu(\theta)\left(U_{1}(t+c \theta)+\tau\left[U_{2}\left(t+a^{*}+c \theta\right)-U_{1}(t+c \theta)\right]\right) .
\end{aligned}
$$

It is apparent that $A(t)$ is an ENN-matrix function, $B(t)$ is nonnegative, and

$$
A(-\infty)=A_{1}, \quad B(-\infty)=B_{1}, \quad A(\infty)=A_{2}, \quad B(\infty)=B_{2} .
$$

By Lemma 3.1 (5) and Lemma 3.3 we have $w(t) \gg 0$ and

$$
w(t)=\gamma_{3} e^{\lambda_{*} t} \eta+o\left(e^{\lambda_{*} t}\right) \quad \text { ase } \quad t \rightarrow \infty .
$$


That is,

$$
\begin{aligned}
& U_{2}\left(t+a^{*}\right) \gg U_{1}(t), \quad t \in \mathbb{R}, \\
& U_{2}\left(t+a^{*}\right)=E_{2}-\gamma_{2} e^{\lambda_{*} a^{*}} e^{\lambda_{*}} \eta+o\left(e^{\lambda_{*} t}\right) \quad \text { as } \quad t \rightarrow \infty
\end{aligned}
$$

with

$$
\gamma_{1}-\gamma_{2} e^{\lambda_{*} a^{*}}=\gamma_{3}>0
$$

Thus, by using (3.7) and the same discussion as above, we easily see that there is a sufficiently small $\epsilon>0$ such that

$$
U_{2}\left(t+a^{*}-\epsilon\right) \geq U_{1}(t), \quad t \in \mathbb{R} .
$$

This contradicts the definition of $a^{*}$, and hence we must have $U_{2}\left(t+a^{*}\right) \equiv U_{1}(t)$. That is, $U_{1}$ is a translation of $U_{2}$.

Case $2 \nu_{1}=\nu_{2}=\nu$ and $k_{1}=k_{2}$.

In this case we must have $\xi_{2}=\delta \xi_{1}$ for some constant $\delta>0$. Without loss of generality, we can suppose $\delta=1$, for otherwise we can consider the translation

$$
\tilde{U}_{2}(t)=U_{2}(t+a)=(-1)^{k} t^{k} e^{\nu t}\left[e^{\nu a} \xi_{2}\right]+o\left(t^{k} e^{\nu t}\right) \text { as } \quad t \rightarrow-\infty,
$$

and choose $a$ such that $e^{\nu a} \delta=1$. It is obvious that $\tilde{U}_{2}(t)$ is a monotone traveling wave connecting $E_{1}$ and $E_{2}$. Now suppose $U_{2} \not \equiv U_{1}$. Then, there is a $t_{0}$ and an integer $j$ such that $U_{1, j}\left(t_{0}\right) \neq U_{2, j}\left(t_{0}\right)$, where $U_{i, j}(t)$ is the $j$ th component of $U_{i}(t)$. For clarity let

$$
U_{1, j}\left(t_{0}\right)>U_{2, j}\left(t_{0}\right)
$$

Let $a^{*}$ be defined as above. Then, in this case, we must have $a^{*}>0$ by (3.8). Also we have

$$
U_{2}\left(t+a^{*}\right) \geq U_{1}(t), \quad t \in \mathbb{R} .
$$

Note that $a^{*}>0$ implies that

$$
U_{2}\left(t+a^{*}\right) \gg U_{1}(t)
$$

for all sufficiently negative $t$. It therefore follows that

$$
U_{2}\left(t+a^{*}\right) \gg U_{1}(t), \quad t \in \mathbb{R} .
$$

By using the same argument as above, we deduce that for some sufficiently small $\epsilon>0$, we have

$$
U_{2}\left(t+a^{*}-\epsilon\right) \gg U_{1}(t), \quad t \in \mathbb{R} .
$$

This again leads to a contradiction. 


\section{References}

[1] A. Berman, R.J. Plemmons. Nonnegative Matrices in the Mathematical Sciences, Classics in Applied Mathematics 9, SIAM, Philadelphia, 1994.

[2] J. Carr, A. Chmaj. Uniqueness of travelling waves for nonlocal monostable equations. Proc. Amer. Math. Soc., 132 (2004), 2433-2439.

[3] T. Faria, W. Huang, J. Wu. Traveling waves for delayed reaction-diffusion equations with global response. Proc. Royal Society A, 462 (2006), 229-261.

[4] W. Huang. Monotonicity of heteroclinic orbits and spectral properties of variational equations for delay differential equations. J. Diff. Equations, 162 (2000), 91-139.

[5] W. Huang, M. Pucket. A note on uniqueness of monotone mono-stable waves for reactiondiffusion equations. Inter. J. Qualitative Theory of Diff. Equations and App., in press (2008).

[6] H. Thieme, X-Q. Zhao. Asymptotic speeds of spread and traveling waves for integral equations and delayed reaction-diffusion models. J. Diff. Equations, 195(2003), 430-470.

[7] V.I. Volpert, V.A. Volpert, V.A. Volpert. Traveling wave solutions of parabolic systems. Translations of Math. Monographs, 140, Amer. Math. Soc., Providence, 1994.

[8] J. Wu. Theory and applications of partial functional differential equations. Applied Mathematical Science, Vol. 119, Springer, Berlin, 1996.

[9] J. Wu, X. Zou. Existence of traveling wave fronts in delayed reaction-diffusion systems via the monotone iteration method. Proc. Amer. Math. Soc., 125 (1997) 2589-2598. 